\title{
More on heart rate variability in obstructive sleep apnea: confusion on a higher level or first step to unravel the cardiovascular mystery of the sleep apnea patient?
}

\author{
Micha T. Maeder
}

Received: 5 October 2013 / Accepted: 8 October 2013 / Published online: 31 October 2013

(C) Springer-Verlag Berlin Heidelberg 2013

There is now a large body of literature indicating that daytime sleepiness is only one clinical aspect of the obstructive sleep apnea syndrome (OSAS) and that OSAS is linked with important cardiovascular conditions including hypertension, atrial fibrillation, stroke, and heart failure [1]. Several studies using different methods (i.e., assessment of muscle sympathetic nerve activity [2], heart rate recovery [3], heart rate variability (HRV) $[2,4]$ ) also have consistently shown that significant OSAS is associated with an autonomic imbalance, i.e., a shift towards sympathetic predominance. It is currently unknown whether such abnormalities of autonomic function are mediators of the adverse cardiovascular outcomes of OSAS patients or whether they simply represent an epiphenomenon and marker of increased cardiovascular risk. Still, sympathetic nervous system overactivity is regarded as a hallmark of significant OSAS as it is a feature shared by OSAS and the associated cardiovascular conditions [5].

In the present issue of Sleep and Breathing, Trimer et al. [6] present another study adding to the evidence that patients with OSAS have disturbed HRV. We cannot avoid asking whether this study is purely confirmatory or whether there are novel aspects. The answer is that the study indeed does confirm the concept of disturbed HRV in OSAS but differs from previous ones with respect to several important aspects. Whereas many previous studies on cardiovascular effects of OSAS have included subjects with severe disease, Trimer et al. focused on subjects with non-severe OSAS. They measured different HRV indices in three groups of subjects: patients with moderate OSAS ( $n=20$, apnea-hypopnea index (AHI) $19.6 \pm 3.7 / \mathrm{h}$ ), patients with mild OSAS ( $n=20$, AHI 8.4

M. T. Maeder $(\bowtie)$

Cardiology Division, Kantonsspital St. Gallen, Rorschacherstrasse

95, 9007 St. Gallen, Switzerland

e-mail: micha.maeder@kssg.ch $\pm 2.5 / \mathrm{h})$, and healthy controls ( $n=18$, AHI $2.8 \pm 1.4 / \mathrm{h})$. All subjects in the study were relatively young (mean age 3540 years), presumably had little comorbidities, and were only moderately overweight, and groups were matched for gender. Thus, the study population was well suited for a pathophysiological study. Second, most previous studies focused on traditional time domain or linear frequency domain parameters including low frequency (LF), high frequency (HF), and LF/HF ratio. In contrast, Trimer et al. also measured a number of novel non-linear HRV parameters including Poincaré plot analysis (SD1 and SD2) and recurrence plot analysis (mean line length, recurrence rate, determinism, and Shannon entropy) to determine whether these novel parameters were more sensitive to identify patients with OSAS compared to traditional linear HRV parameters. Importantly, only apnea-free samples rather than signals from a full night were considered for analysis. Thus, the chronic effect of OSAS rather than the acute effect of a cycle of apnea and arousal was assessed. Third, measurements were obtained during different sleep stages, i.e., rapid eye movement (REM) sleep and non-REM sleep, since previous work had suggested that this might provide additional insights into the balance of sympathetic and parasympathetic drive to the heart [7].

The authors found higher LF, LF/HF ratio, and determinism (a novel non-linear HRV parameter) and lower HF in patients with moderate OSAS compared to controls, and this was true for both REM sleep and stage 2 non-REM sleep. In contrast, there were no significant differences in LF, $\mathrm{HF}$, and the LF/HF ratio between controls and patients with mild OSAS, and there were no differences in non-linear HRV parameters other than determinism between all three groups. Thus, this study tells us that in contrast to the authors' expectation, non-linear HRV parameters are not better than traditional frequency domain parameters for the detection of autonomic abnormalities in patients with a relatively early stage of the disease. 
What else could this study tell us? A landmark study had suggested (although a randomized trial has never been performed) that subjects with untreated severe OSAS had an increased risk of cardiovascular death, whereas this was not the case in patients with untreated mild to moderate disease [8]. A reasonable interpretation of this finding could be that subjects with severe OSAS should be treated with continuous positive airway pressure not only to improve symptoms of daytime sleepiness but also to reduce cardiovascular risk, but less symptomatic subjects with milder disease do not necessarily need treatment. The present study showed however that also subjects with moderate OSAS clearly had alterations in autonomic tone, i.e., a sympathetic predominance suggesting an increased cardiovascular risk in patients with non-severe OSAS which raises the question about the need for treatment also in these patients.

And what do the results mean with regard to subjects with mild disease? Although there were no differences in classical frequency domain parameters and non-linear HRV parameters between patients with mild OSAS and controls, the comparison of HRV parameters between sleep stages revealed another interesting finding: while in healthy controls and patients with moderate OSAS there were significantly higher LF and the LF/HF ratio and lower HF during REM compared to stage 2 sleep, this was not the case in patients with mild OSAS. This finding was interpreted by the authors as a possible early sign of autonomic dysfunction in patients with mild OSAS. Although inspection of Figure 1 in the manuscript by Trimer et al. [6] suggests that this lack of difference in HRV indices between REM and non-REM stage 2 sleep might simply have been due to lack of power, this is an interesting idea which deserves further studies. Thus, the present study tells us that HRV analysis has the potential to detect subtle changes in autonomic tone also in patients with moderate OSAS and maybe even in those with mild disease.

Where are we going from here? A large number of studies on HRV in OSAS have been published, and there are still no clinical applications. Still, one scenario is the following: OSAS is associated with an increased risk of sudden cardiac death [9], and sudden deaths in OSAS patients frequently occur during sleep [10]. There is currently no tool to identify OSAS patients at risk for sudden cardiac death. The risk of sudden death in OSAS has been shown to depend on the severity of OSAS as expressed by the AHI as well as the degree of nocturnal desaturation [9]. A very recent provocative study has revealed that HRV abnormalities such as high LF and low HF in patients with sleep-disordered breathing are related to the degree of hypoxemia, whereas the mechanism leading to hypoxia, i.e., obstructive events versus central events are less important [11]. One might therefore speculate that HRV analysis probably combined with classical measures of OSAS severity might become a tool to identify OSAS patients at risk for sudden cardiac death. However, until this becomes a reality, there is a long way to go: First, HRV analysis, which can be a cumbersome undertaking, must be simple and reproducible. Then, large prospective studies on the association between HRV and outcomes in OSAS are required, the independent information of HRV compared to classical descriptors of OSAS severity will have to be described, potential mediators of sudden death in those with pathological HRV will have to be identified (e.g., silent coronary artery disease, left ventricular dysfunction, and scarring, respectively), and the role of HRV to tailor treatment of OSAS in general and to prevent sudden cardiac death in particular needs to be established. Given the magnitude of the problem, such efforts seem to be justified however.

\section{References}

1. Bradley TD, Floras JS (2009) Obstructive sleep apnoea and its cardiovascular consequences. Lancet 373:82-93

2. Narkiewicz K, Montano N, Cogliati C, van de Borne PJ, Dyken ME, Somers VK (1998) Altered cardiovascular variability in obstructive sleep apnea. Circulation 98:1071-1077

3. Maeder MT, Munzer T, Rickli H, Schoch OD, Korte W, Hurny C, Ammann P (2008) Association between heart rate recovery and severity of obstructive sleep apnea syndrome. Sleep Med 9:753-761

4. Roche F, Gaspoz JM, Court-Fortune I, Minini P, Pichot V, Duverney D, Costes F, Lacour JR, Barthelemy JC (1999) Screening of obstructive sleep apnea syndrome by heart rate variability analysis. Circulation 100:1411-1415

5. Levy P, Ryan S, Oldenburg O, Parati G (2013) Sleep apnoea and the heart. Eur Respir Rev 22:333-352

6. Trimer R, Mendes RG, Costa FSM, Sampaio LMM, Delfino Jr A, Arena R, Aletti F, Ferrario M, Borghi-Silva A (2013) Is there a chronic sleep-stage dependent linear and nonlinear cardiac autonomic impairment in obstructive sleep apnea? Sleep Breath. doi:10.1007/s11325-013-0900-x

7. Cabiddu R, Cerutti S, Viardot G, Werner S, Bianchi AM (2012) Modulation of the sympatho-vagal balance during sleep: frequency domain study of heart rate variability and respiration. Front Physiol 3:45

8. Marin JM, Carrizo SJ, Vicente E, Agusti AG (2005) Long-term cardiovascular outcomes in men with obstructive sleep apnoeahypopnoea with or without treatment with continuous positive airway pressure: an observational study. Lancet 365:1046-1053

9. Gami AS, Olson EJ, Shen WK, Wright RS, Ballman KV, Hodge DO, Herges RM, Howard DE, Somers VK (2013) Obstructive sleep apnea and the risk of sudden cardiac death: a longitudinal study of 10,701 adults. J Am Coll Cardiol 62:610-616

10. Gami AS, Howard DE, Olson EJ, Somers VK (2005) Day-night pattern of sudden death in obstructive sleep apnea. N Engl J Med 352: 1206-1214

11. Palma JA, Urrestarazu E, Lopez-Azcarate J, Alegre M, Fernandez S, Artieda J, Iriarte J (2013) Increased sympathetic and decreased parasympathetic cardiac tone in patients with sleep related alveolar hypoventilation. Sleep 36:933-940 